Please do not remove this page

RMIT

UNIVERSITY

\title{
Performance evaluation of a linear predictor frequency estimator for mobile flat fading wireless channels
}

Sithamparanathan, Kandeepan; Rohani, Bijan; Caldera, Manora

https://researchrepository.rmit.edu.au/esploro/outputs/9921858203301341/filesAndLinks?institution=61RMIT_INST\&index=null

Sithamparanathan, K., Rohani, B., \& Caldera, M. (2009). Performance evaluation of a linear predictor frequency estimator for mobile flat fading wireless channels. IEEE Transactions on Vehicular Technology, PP(99), 1-6. https://doi.org/10.1109/TVT.2008.2004556

Published Version: https://doi.org/10.1109/TVT.2008.2004556

Repository homepage: https://researchrepository.rmit.edu.au (C) IEEE

Downloaded On 2023/04/26 19:56:49 +1000 
Thank you for downloading this document from the RMIT Research Repository.

The RMIT Research Repository is an open access database showcasing the research outputs of RMIT University researchers.

RMIT Research Repository: http://researchbank.rmit.edu.au/

\section{Citation:}

Sithamparanathan, K, Rohani, B and Caldera, M 2009, 'Performance evaluation of a linear predictor frequency estimator for mobile flat fading wireless channels', IEEE Transactions on Vehicular Technology, vol. PP, no. 99, pp. 1-6.

See this record in the RMIT Research Repository at:

http://researchbank.rmit.edu.au/view/rmit:12657

Version: Accepted Manuscript

Copyright Statement: (C) IEEE

Link to Published Version:

http://dx.doi.org/10.1109/TVT.2008.2004556

\section{PLEASE DO NOT REMOVE THIS PAGE}




\title{
Performance Evaluation of a Linear Predictor Frequency Estimator for Mobile Flat Fading Wireless Channels
}

\author{
* Sithamparanathan Kandeepan, Member, IEEE, Bijan Rohani, Manora Caldera, \\ * Create-Net International Research Centre, via alla Cascata 56/D, 38100 Povo, Trento, Italy \\ kandeepan@ieee.org \\ Ph: +39 0461408400 ext: 405, Fax: +390461421157
}

\begin{abstract}
A well known frequency estimation algorithm using linear prediction method is analysed for flat fading wireless channels. The estimator outputs are statistically analysed and its jitter performances are compared with the non-fading case and the Cramer-Rao bound. We provide a closed form solution for the distribution and the variance of the frequency estimates under fading conditions by making valid assumptions. We also verify the theoretical model using simulations. Analysis shows that the variance of the estimates for flat fading channels reaches a threshold point and increasing the transmit power does not necessarily improve the performances any further.
\end{abstract}

\section{Index Terms}

linear predictive frequency estimator, mobile flat fading channel, frequency synchronization, frequency distribution.

\section{INTRODUCTION}

Synchronization of the carrier frequency is a well know problem in communications and many techniques exist in literature addressing this problem [10],[13]-[26]. Frequency synchronization for wireless communications require estimation of frequencies in fading environments. Extensive literature exist on frequency estimation techniques for additive white Gaussian noise only channels and a few on fading channels [1]-[10]. The analysis of well known frequency estimators such as the maximum likelihood (ML) estimator [9],[10], Kay's estimator [8], the periodogram search method [9], sub-space methods [7], linear predictor based methods [4]-[6], DFT based methods [10] etc, are well treated for additive Gaussian noise only channel. In this paper, we present the performance measures of a well known frequency estimation technique based on linear prediction method [4]-[6] for flat fading wireless channels [27]. In [1]-[2],

\footnotetext{
Manuscript revised and submitted July 21, 2008, to the IEEE Transactions on Vehicular Technology.
}

This research was funded by the National ICT Australia (NICTA) under the Last Metre Problem (LaMP) project from $2004-2007$. Dr. Kandeepan is currently with Create-Net and the Department of Information Engineering at the Australian National University. He was formerly with the NICTA in Canberra. Dr. Rohani and Dr. Caldera are with the WATRI. National ICT Australia is funded through the Australian Government's Backing Australia's Ability initiative, in part through the Australian Research Council 
the authors have investigated ML based frequency estimation techniques and modified versions of the techniques for fading channels, they have also presented the variance of the estimates. However, the analysis and results in [1]-[2] are based on linear models assuming the estimates to have a Gaussian distribution. In [1], a linear predictor based frequency estimator has been treated for fading channels assuming linearity in the signal plus noise model. In our analysis we consider the nonlinear effects of the arctan function used in the estimator and derive a precise closed form solution for the distribution of the frequency estimates, by making valid assumptions and invoking the central limit theorem appropriately. We also determine the variance associated with the frequency estimates and derive an approximated closed form solution.

The analyses on frequency estimators in [1]-[3] do not present the distribution of the estimates. Knowing the distribution of the estimates help system engineers and researchers working in the field of advanced receiver designs and synchronisation to predict the bit error probabilities of the communication link. Identifying the statistical distribution of the frequency estimates is the key to predict bit error rate (BER) degradation caused due to synchronization errors. The distribution of the frequency estimates is used to quantify the BER of a practical digital communication system against frequency jitter and signal to noise ratio. Especially, in OFDM (Orthogonal Frequency Division Multiplexed) systems, the frequency errors will cause inter symbol interferences [28] and such errors can be quantified with the knowledge of the statistical distribution of the frequency estimates. The BER analysis of such systems are treated on a case by case basis depending on the type of modulation, coding and transmission techniques used, and is beyond the scope of this paper. Here we only provide the solution to the distribution of the frequency estimates based on the linear predictor estimator.

\section{Signal And Channel Model}

The channel model considered here is a time correlated flat fading Rayleigh channel. The time domain correlation depends on the mobility of the transmitter and the receiver units and the surroundings. The $n^{\text {th }}$ sample of the complex sinusoidal signal received through a correlated Rayleigh fading channel in the presence of additive white Gaussian noise (AWGN) is given by,

$$
r[n]=\gamma[n] \operatorname{Aexp}(j \Omega n+j \phi)+\eta(n)
$$

where, $A$ is the signal amplitude, $\phi$ is a slowly varying random phase offset with a uniform distribution between $[-\pi,+\pi]$ which is a constant within the signal observation time, and $\Omega$ is the discrete frequency 
in radians that needs to be estimated. Where, $\Omega=2 \pi f / f_{s}, f_{s}$ is the sampling frequency, and $f$ is the analog frequency in Hz. The frequency $f$ includes the Doppler frequency drift and the local oscillator drifts between the transmitter and the receiver. The channel coefficient $\gamma[n]$ is a zero mean complex Gaussian process with a variance of $2 \sigma_{a}^{2}$ that describes the multiplicative distortion of the channel,

$$
\gamma[n]=\gamma_{i}[n]+j \gamma_{q}[n]
$$

It should be noted that $\gamma_{i}[n]$ and $\gamma_{q}[n]$ are individually correlated with time and independent to each other. The envelope of the channel $|\gamma[n]|=\sqrt{\gamma_{i}[n]^{2}+\gamma_{q}[n]^{2}}$ is a Rayleigh distributed random variable. The time correlation function describing the in-phase and quadrature components of the fading channel is modeled as [11],

$$
R_{\gamma_{i}}(n)=R_{\gamma_{q}}(n)=R(n)=\exp \left(-\frac{|v||n|}{d_{c}} \frac{\mid n}{f_{s}} \ln (2)\right)
$$

where $v$ is the velocity of the receiver with respect to the transmitter and $d_{c}$ is the de-correlation distance that defines the surroundings of the wireless channel. Typically, for an indoor channel, $d_{c}=5 \mathrm{~m}$ and for an outdoor channel $d_{c}=20 \mathrm{~m}$ [11]. In other words for a given $f_{s}$ and $v$, if $d_{c}=5 \mathrm{~m}$, then samples taken at distances greater than $5 \mathrm{~m}$ intervals in the wireless channel are highly uncorrelated, and the samples taken at distances less than $5 \mathrm{~m}$ intervals are highly correlated. In this paper we analyse the frequency estimator performances for both slow and fast flat-fading channels. Moreover, $\eta[n]$ is an i.i.d. AWGN component that can be expressed as

$$
\eta[n]=\eta_{i}[n]+j \eta_{q}[n]
$$

where $\eta_{i}[n]$ and $\eta_{q}[n]$ are two independent zero mean Gaussian random processes each with a variance of $\sigma^{2}=N_{0} f_{s}$, with a double sided power spectral density of $N_{0}$ (Watts/Hz). Here, we define the expected signal to noise ratio $(S N R)$ of the received signal as, $\Upsilon=A^{2} \sigma_{a}^{2} / \sigma^{2}$.

\section{FREQUENCY Estimator}

The linear predictive frequency estimator, which we here refer to as the single-delay multiplication (SD-M) based frequency estimator, for $L$ samples is given by [4]-[6],

$$
\hat{\Omega}=\arctan \left\{\sum_{n=1}^{L} r[n] r^{*}[n-1]\right\}
$$

The received signal is multiplied by its delayed conjugate and averaged over $L$ samples before taking the arctan function to perform an estimate on the frequency. In the noiseless case the estimator output directly 
computes the discrete frequency of the signal $\Omega$ as given in (5). However, when there is additive noise present together with fading the performances of the estimator degrades. In the following sections we analyse the estimator and find a closed form expression for the distribution of $\hat{\Omega}$, and its related statistics.

\section{ANALysis OF ThE FreQUenCy Estimator}

Let us examine the SD-M algorithm step by step. Let,

$$
\varepsilon[n]=r[n] r^{*}[n-1]
$$

Considering the received signal model in (1), (6) becomes,

$$
\varepsilon[n]=S_{0}[n]+P_{1}(n)+P_{2}(n)+P_{3}(n)
$$

where,

$$
\begin{aligned}
& S_{0}[n]=\gamma[n] \gamma^{*}[n-1] A^{2} \exp (j \Omega), P_{1}[n]=A \exp (j \Omega n+j \phi) \gamma[n] \eta^{*}[n-1] \\
& P_{2}[n]=A \exp (-j \Omega(n-1)-j \phi) \gamma^{*}[n-1] \eta[n], \text { and } P_{3}[n]=\eta[n] \eta^{*}[n-1]
\end{aligned}
$$

We analyse the statistical behavior of each of the above terms in (8) separately.

\section{A. Unbiased Estimator}

To start with, let us analyse whether the estimator is unbiased or not when operated under fading conditions. The expected value of $\varepsilon[n]$ is given by

$$
E\{\varepsilon[n]\}=E\left\{S_{0}[n]\right\}+E\left\{P_{1}[n]\right\}+E\left\{P_{2}[n]\right\}+E\left\{P_{3}[n]\right\}
$$

then, with the definitions of the noise and the channel models, it can be easily found that in (9), $E\left\{P_{1}[n]\right\}=$ $0, E\left\{P_{2}[n]\right\}=0$ and $E\left\{P_{3}[n]\right\}=0$, therefore,

$$
E\{\varepsilon[n]\}=E\left\{S_{0}[n]\right\}=2 R(1) A^{2} \exp (j \Omega)
$$

Without loss of generality, we could now argue that the phase of $E\left\{S_{0}[n]\right\}$, which is the estimate from the SD-M frequency estimator is the same as the original frequency $\Omega$. Hence the estimator is unbiased under fading conditions only if the channel is correlated (i.e. $R(1) \neq 0$ ). 


\section{B. Noise Analysis}

Let us consider the second step of the SD-M algorithm, i.e., the summation over $L$ samples.

$$
Z_{L}=S_{L}+\chi_{1}+\chi_{2}+\chi_{3}
$$

where,

$$
S_{L}=\sum_{n=1}^{L} S_{0}[n], \chi_{1}=\sum_{n=1}^{L} P_{1}[n], \chi_{2}=\sum_{n=1}^{L} P_{2}[n], \chi_{3}=\sum_{n=1}^{L} P_{3}[n]
$$

In the process of our analysis we treat each of the terms in (11) separately for its statistical behavior and combine the analysis at the end. Using the summation operation over $L$ samples and together with other sufficient conditions [12] we can invoke the central limit theorem in (12) to simplify the analysis. The analysis of $\chi_{3}$ and its corresponding statistical properties are readily available in [6]. From [6], $\chi_{3}$ follows a complex Gaussian distribution with zero mean and a variance of $2 \sigma^{2} \chi_{3}=4 L \sigma^{4}$. Further, it also can be shown that $\chi_{3}$ is an independent process. Therefore, the distribution of $\chi_{3}$ is given by $f_{\chi_{3}}\left(\chi_{3}\right) \sim C N\left(0,2 \sigma^{2} \chi_{3}\right)$, where, $C N(a, b)$ denotes a complex Gaussian distribution with mean $a$ and variance $b$. The other two noise-channel cross product terms $\chi_{1}$ and $\chi_{2}$ in (11) have similar statistical properties as each other. Therefore, we analyse $\chi_{1}$ and then extend the results to $\chi_{2}$. Let us consider

$$
\chi_{1}=\sum_{n=1}^{L} P_{1}[n]=\sum_{n=1}^{L} P_{11}[n] \gamma^{*}[n-1]
$$

where

$$
P_{11}[n]=A \exp (-j \Omega(n-1)-j \phi) \eta[n]
$$

Again from [6], the distribution of the complex process $P_{11}[n]$ follows a Gaussian distribution with zero mean and a variance of $2 A^{2} \sigma^{2}$, that is $f_{P_{11}}\left(P_{11}\right) \sim C N\left(0,2 A^{2} \sigma^{2}\right)$. Now, given that $P_{11}$ is Gaussian and $\gamma^{*}[n-1]$ is Gaussian, then similar to $\chi_{3}, \chi_{1}$ also follows a Gaussian distribution with zero mean and a variance of $2 \sigma_{\chi_{1}}^{2}=4 L A^{2} \sigma_{a}^{2} \sigma^{2}$. Therefore, $f_{\chi_{1}}\left(\chi_{1}\right) \sim C N\left(0,2 \sigma^{2}{ }_{\chi_{1}}\right)$. Here, the expression for the variance $\sigma^{2}{ }_{\chi_{1}}$ states that increasing the transmit signal power $\left(A^{2}\right)$ will increase the noise power within the estimator. This is rather misleading to the reader. It should be noted that increasing the transmit signal power also increases the signal component of our interest in (11) within the estimator, therefore the correct way to interpret the results is to consider the overall signal to noise ratio rather than the noise power itself. Further, since $\gamma^{*}[n-1]$ is a correlated random process, it is also important for us to know whether $\chi_{1}$ is correlated. To verify this, consider the following: Let $\xi[n]=P_{11}[n] \gamma^{*}[n-1]$, and since $P_{11}$ is an 
independent process, then the auto-correlation function of $\xi[n]$ is given by

$$
R_{\xi}(m)=E\left[P_{11}[n] P_{11}[n-m]\right] E\left[\gamma^{*}[n-1] \gamma^{*}[n-1-m]\right]
$$

and since $E\left[P_{11}[n] P_{11}[n-m]\right]=0$, the autocorrelation function $R_{\xi}(m)$ becomes zero. Therefore, we can say that $\chi_{1}$ is an independent random process. The second noise-channel cross product term $\chi_{2}$ also has similar properties as $\chi_{1}$ and hence the distribution is given by $f_{\chi_{2}}\left(\chi_{2}\right) \sim C N\left(0,2 \sigma_{\chi_{2}}^{2}\right)$, where $\sigma_{\chi_{2}}^{2}=\sigma_{\chi_{1}}^{2}$. Therefore, all three noise-channel cross product terms $\chi_{1}, \chi_{2}$, and $\chi_{3}$ can be modeled as Gaussian. Now, let us define

$$
\chi=\chi_{1}+\chi_{2}+\chi_{3}
$$

Then, by combining the distributions of $\chi_{1}, \chi_{2}$ and $\chi_{3}, \chi$ also becomes a complex Gaussian process with zero mean and a variance of $2 \sigma_{\chi}^{2}=2 \sigma_{\chi_{1}}^{2}+2 \sigma_{\chi_{2}}^{2}+2 \sigma_{\chi_{3}}^{2}$. That is

$$
f_{\chi}(\chi) \sim C N\left(0,2 \sigma^{2}{ }_{\chi}\right)
$$

Therefore, $Z_{L}$ becomes

$$
Z_{L}=S_{L}+\chi
$$

Now let us analyse $S_{L}$. From (12), we can write $S_{L}$ as

$$
S_{L}=\sum_{n=1}^{L} \gamma[n] \gamma^{*}[n-1] A^{2} \exp [j \Omega]
$$

Here, we recognise that $S_{L}$ is similar to $\chi_{3}$ and without much effort could state that $S_{L}$ follows a Gaussian distribution with a variance of $2 \sigma_{S_{L}}^{2}=4 A^{4} \sigma_{a}^{4} L$. However, the fact that $\gamma[n]$ is a correlated process, leads to a non-zero mean for $S_{L}$. From (10), we identify that the real and the imaginary parts of $S_{L}$ have mean values given by $m_{1}=2 A^{2} L R_{(1)} \cos (\Omega)$ and $m_{2}=2 A^{2} L R_{(1)} \sin (\Omega)$ respectively. Therefore, we can write the distribution of $S_{L}$ as $f_{S_{L}}\left(S_{L}\right) \sim C N\left(m_{1}+j m_{2}, 2 \sigma_{S_{L}}^{2}\right)$. Further, for convenience, we can rewrite $S_{L}$ as

$$
S_{L}=S_{m}+S_{n}
$$

where, $S_{m}=2 R_{(1)} A^{2} \exp (j \Omega)$ and $S_{n}$ is a zero mean complex Gaussian process with a variance of $2 \sigma_{S_{L}}^{2}$. Note that $S_{n}$ is the noise term introduced by the fading channel which is independent to the additive noise term. This term $S_{n}$ leads to a threshold effect in the jitter performance of the estimator which we analyse in later sections. The term $S_{m}$ is our main signal component that contains the parameter of our interest, which is the discrete frequency to be estimated. The rest of the terms such as $S_{n}$ and $\chi$ are all treated as 
noise terms within the estimator. Therefore, we can rewrite $Z_{L}$ as,

$$
Z_{L}=Z_{1}+j Z_{2}=S_{m}+\left(S_{n}+\chi\right)
$$

Clearly, $Z_{L}$ follows a complex Gaussian process given by

$$
Z_{1} \sim N\left(m_{1}, \sigma_{S_{n}}^{2}+\sigma_{\chi}^{2}\right) \text { and, } Z_{2} \sim N\left(m_{2}, \sigma_{S_{n}}^{2}+\sigma_{\chi}^{2}\right)
$$

Finally, the next step of the SD-M algorithm which is the arctan function on $Z_{L}$ is considered, and is treated in the following section.

\section{Distribution of the Frequency Estimates}

The arctan function is a nonlinear operation which is the final step of the SD-M estimator algorithm. We treat this as a transformation of random variable given by the nonlinear transformation,

$$
\widehat{\Omega}=\arctan \left\{\frac{Z_{2}}{Z_{1}}\right\}
$$

Knowing the joint distribution $f_{z_{1} z_{2}}\left(z_{1}, z_{2}\right)$, of $Z_{1}$ and $Z_{2}$, the distribution of $\hat{\Omega}$ is then derived by,

$$
f_{\hat{\Omega}}(\hat{\Omega})=\int_{-\infty}^{\infty} f_{z_{1} z_{2}}\left(z_{1}, z_{2}\right)\left|J\left(z_{1}, z_{2}\right)\right|^{-1} d z_{1}
$$

where, $z_{2}=z_{1} \tan (\hat{\Omega})$, and

$$
\left|J\left(z_{1}, z_{2}\right)\right|=\left|\begin{array}{cc}
\frac{\partial z_{1}}{\partial \varphi} & \frac{\partial z_{1}}{\partial z_{2}} \\
0 & 1
\end{array}\right|^{-1}
$$

The solution is given in equation (26). The equation is solved using integration by parts and identifying the existence of the complementary error function which is expressed using the $Q($.$) function as given in$ (28). Since the arctan function is discontinuous at the points $-\pi / 2$ and $\pi / 2$ the resulting integration takes up three different ranges $[-\pi,-\pi / 2),[-\pi / 2, \pi / 2]$ and $(\pi / 2, \pi]$. By solving the integration in (24), we can obtain a closed form solution for the frequency estimates $\hat{\Omega}$, which is given in (26), where $\sigma_{Z_{L}}^{2}=\sigma_{S_{n}}^{2}+\sigma_{\chi}^{2}$. In section $\mathrm{V}$ we provide simulation results to verify the theoretical work provided in this section. 


$$
\begin{gathered}
f_{\hat{\Omega}}(\hat{\Omega})= \begin{cases}\exp (-\varsigma / 2)\left[\frac{1}{2 \pi}-\exp \left(\frac{\Gamma_{1}}{2}\right)\left(\frac{\Gamma_{1}}{2 \pi}\right)^{\frac{1}{2}} Q\left(\sqrt{\Gamma_{1}}\right)\right] & \text { for } \pi / 2<\hat{\Omega} \leq \pi \\
\exp (-\varsigma / 2)\left[\frac{1}{2 \pi}+\exp \left(\frac{\Gamma}{2}\right)\left(\frac{\Gamma}{2 \pi}\right)^{\frac{1}{2}}(1-Q(\sqrt{\Gamma}))\right] & \text { for }-\pi / 2<\hat{\Omega} \leq \pi / 2 \\
\exp (-\varsigma / 2)\left[\frac{1}{2 \pi}-\exp \left(\frac{\Gamma_{2}}{2}\right)\left(\frac{\Gamma_{2}}{2 \pi}\right)^{\frac{1}{2}} Q\left(\sqrt{\Gamma_{2}}\right)\right] & \text { for }-\pi<\hat{\Omega} \leq-\pi / 2\end{cases} \\
\text { where, } \varsigma=\frac{m_{1}^{2}+m_{2}^{2}}{\sigma_{Z_{L}}^{2}}, \Gamma=\frac{\left[\tan (\hat{\Omega}) m_{1}+m_{2}\right]^{2}}{\left[1+\tan ^{2}(\hat{\Omega})\right] \sigma_{Z_{L}}^{2}} \Gamma_{1}=\frac{\left[\tan (\pi+\hat{\Omega}) m_{1}+m_{2}\right]^{2}}{\left[1+\tan ^{2}(\pi+\hat{\Omega})\right] \sigma_{Z_{L}}^{2}} \\
\Gamma_{2}=\frac{\left[\tan (-\pi+\hat{\Omega}) m_{1}+m_{2}\right]^{2}}{\left[1+\tan ^{2}(-\pi+\hat{\Omega})\right] \sigma_{Z_{L}}^{2}} \text { and }, Q(x)=\frac{1}{2 \pi} \int_{x}^{\infty} \exp \left(-u^{2} / 2\right) d u
\end{gathered}
$$

\section{Variance of the Frequency Estimates}

The variance of the frequency estimates is computed from the distribution obtained from the previous section. The variance of the estimates is useful to quantify the synchronisation performances. In general, large frequency variance gives poor synchronisation performances. Here we provide an approximated closed form solution for the variance of the frequency estimates and in the next section we show that the approximated expression matches closely with the simulation results. The variance is computed by solving the integration in (29),

$$
\sigma_{\hat{\Omega}}^{2}=\int_{-\infty}^{\infty}(\hat{\Omega}-E(\hat{\Omega}))^{2} f_{\hat{\Omega}}(\hat{\Omega}) d \hat{\Omega}
$$

Considering the functional behavior of (26), we perform a low $S N R$ approximation and a high $S N R$ approximation to determine the frequency variance. At low $S N R$, as the tails of the distribution rise with decreasing $S N R$, we use the the Taylor series expansion and its first two terms to solve the integral in (29). Analysis show that the low $S N R$ approximation is valid for $\varsigma<5 \mathrm{~dB}$. Therefore, the variance of the frequency estimates for $\varsigma<5 \mathrm{~dB}$ is approximately given by,

$$
\sigma_{\hat{\Omega}}^{2}=\exp (-\varsigma / 2)\left[\frac{\pi^{2}}{3}+\sqrt{\frac{\varsigma}{2 \pi}}\left[\frac{\pi^{2}(3+\varsigma)}{12}-\left(2+\frac{13 \varsigma}{18}\right)\right]\right]
$$

where, from (27),

$$
\varsigma=\frac{2 L R_{(1)}^{2} \Upsilon^{2}}{(1+\Upsilon)^{2}}
$$




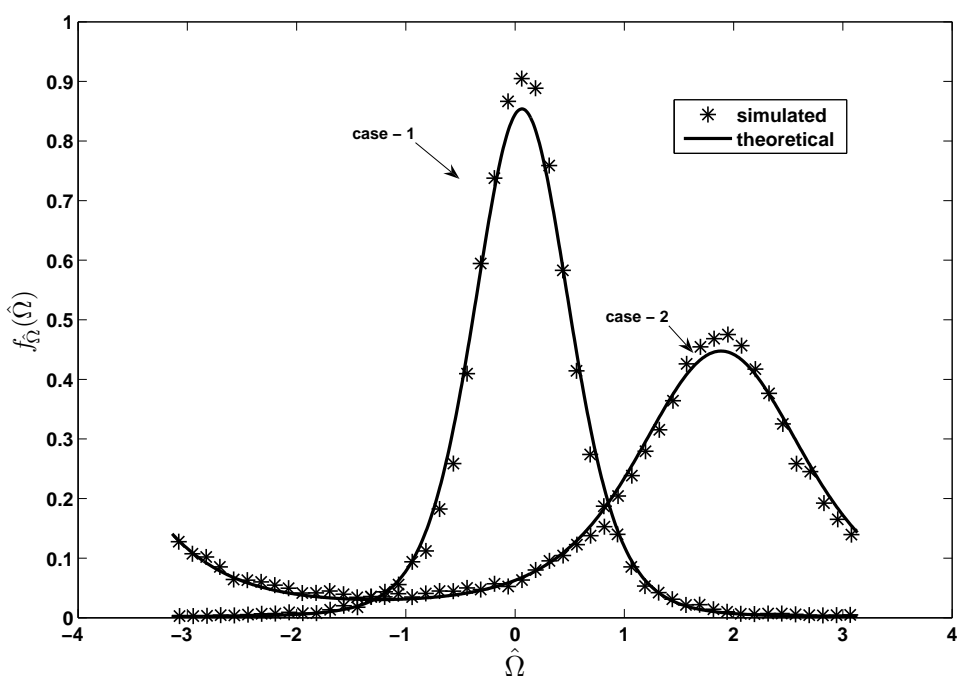

Fig. 1. pdf of the frequency estimate $\hat{\Omega}$, case-1: $S N R \Upsilon=-3 \mathrm{~dB}, \mathrm{~L}=60, \mathrm{f}=20 \mathrm{~Hz}, f_{s}=2000 \mathrm{~Hz}$, case-2: $S N R \Upsilon=-6 \mathrm{~dB}, \mathrm{~L}=40$, $\mathrm{f}=600 \mathrm{~Hz}, f_{s}=2000 \mathrm{~Hz}$

We also observe that for $\Upsilon=-\infty d B$ the distribution in (26) becomes a uniform distribution between $-\pi$ and $\pi$ and the variance takes a value of $\sigma_{\hat{\Omega}}^{2}=\pi^{2} / 3$. This is due to the nonlinear modulo- $2 \pi$ nature of the arctan function. The high $S N R$ approximation for the variance is given by (for $\varsigma \geq 5 \mathrm{~dB}$ ),

$$
\sigma_{\hat{\Omega}}^{2}=\frac{1}{\varsigma}
$$

We observe that the variance of the estimate depends on the channel correlation coefficient $R_{(1)}$. As expected, the variance of the estimates reduces for highly correlated channels.

\section{Simulation Results And Performance Analysis}

The algorithm was simulated in Matlab and results were obtained for various channel conditions. In this section we verify the theoretical claims provided in the previous sections by means of simulations. The distribution for the frequency estimates $\hat{\Omega}$ is shown in Fig-1, together with the simulated results. The results are shown for two different scenarios. From the figure we see a close match of the simulated results with the theory given in equation (26). The variance of the frequency is given in Fig-2. The simulated results are matched well with the approximated expressions for the the variance as given in (30) and (32). The figure also shows how the variance of the estimate reaches a constant lower bound as the $S N R$ is increased.

It is quite important to know how the frequency estimates degrade under worsening channel conditions. We analyse the performance of the estimator with varying number of samples $L$, input signal to noise ratio 


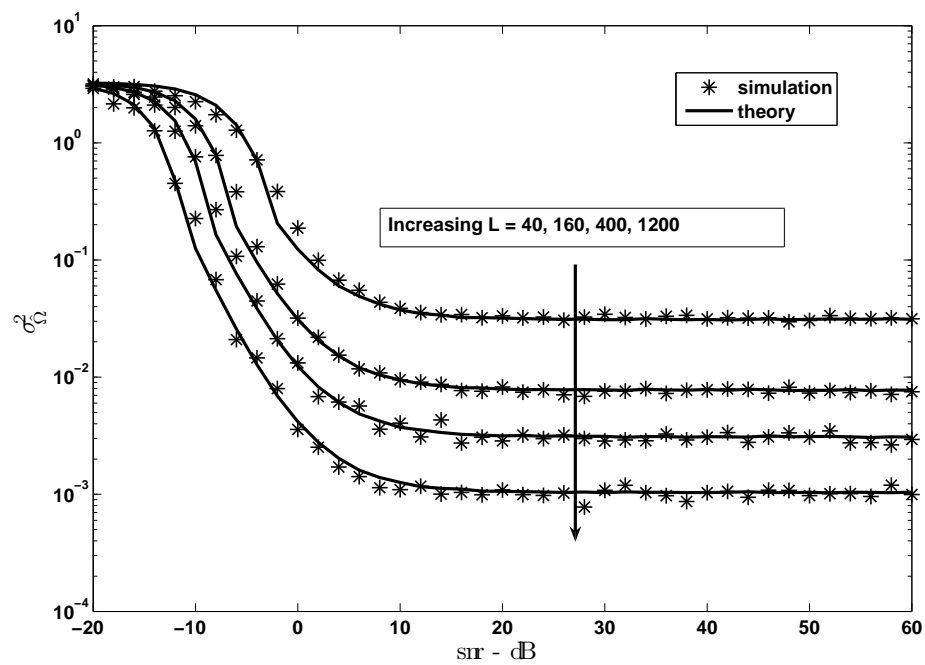

Fig. 2. $\quad$ variance of the frequency estimates, $f=20 H z, f_{s}=2 k H z, d_{c}=0.01, v=1.4$

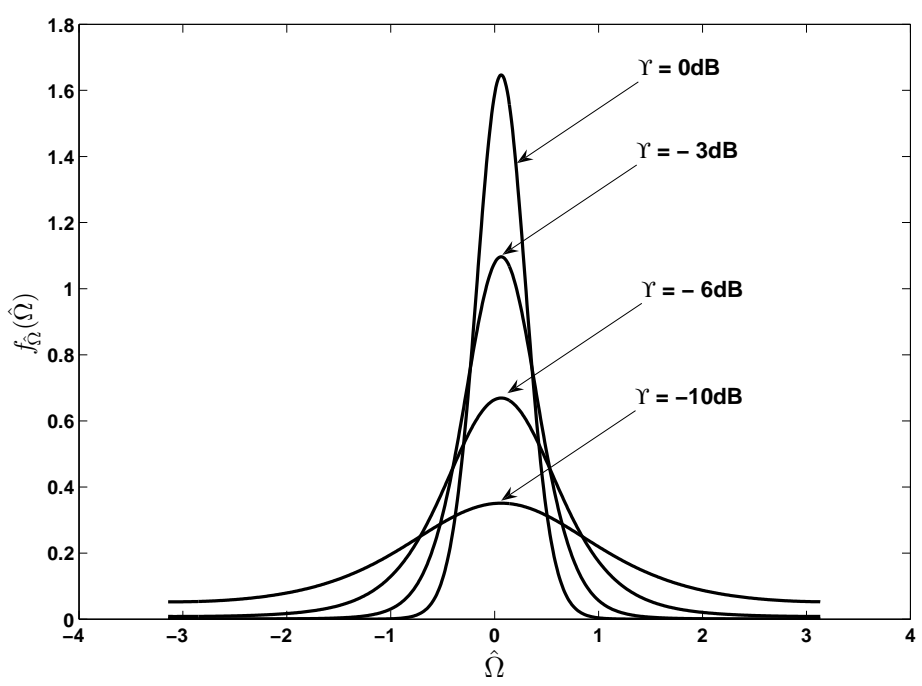

Fig. 3. pdf of $\hat{\Omega}, L=100, f=20 H z, f_{s}=2 k H z, d_{c}=1, v=1.4$

$S N R$, and different channel conditions with different correlation values. The variation of $f_{\hat{\Omega}}(\hat{\Omega})$ with $S N R$ is shown in Fig-3. As expected the distribution gets narrower and peaky when $S N R$ is increased, while the other parameters remain unchanged. However, when the $S N R$ is further increased the distribution $f_{\hat{\Omega}}(\hat{\Omega})$ reaches a point where the additive Gaussian noise becomes less dominant compared to the disturbances caused by the channel conditions (i.e. $S_{n}$ becomes more dominant than $\chi$ ), and the distribution $f_{\hat{\Omega}}(\hat{\Omega})$ shows no further improvement afterwards. This can be more clearly seen in the frequency variance plot of the estimator in Fig-2. Fig-4 shows the improvement in the distribution $f_{\hat{\Omega}}(\hat{\Omega})$ when the number of samples per estimate $L$ is increased. The variance of the frequency estimates is also shown in Fig-5 for 


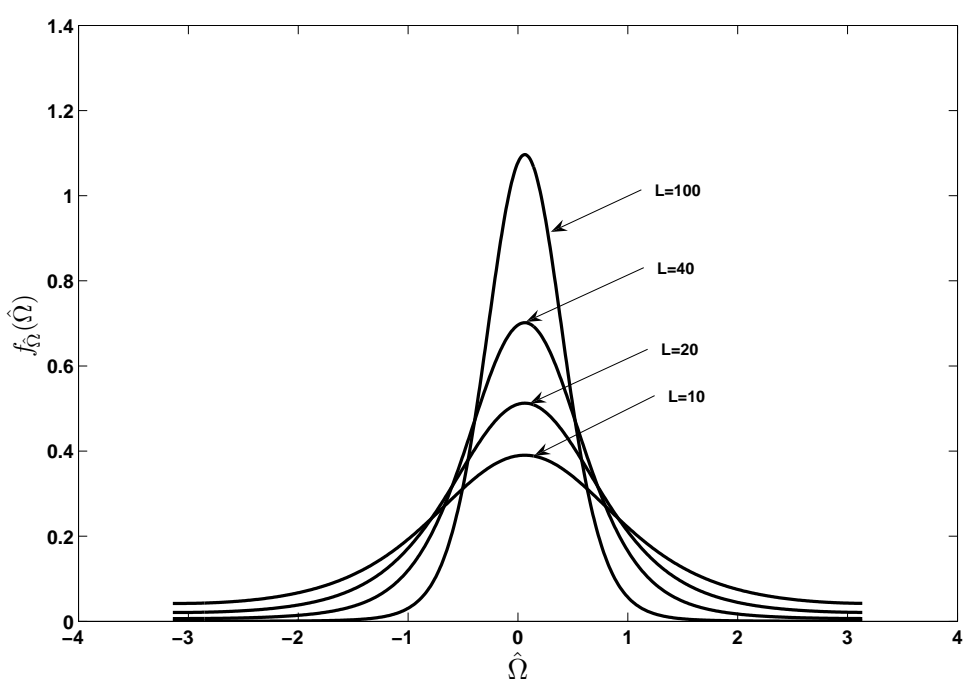

Fig. 4. $\quad$ pdf of $\hat{\Omega}, S N R \Upsilon=-3 d B, f=20 H z, f_{s}=2 k H z, d_{c}=1, v=1.4$

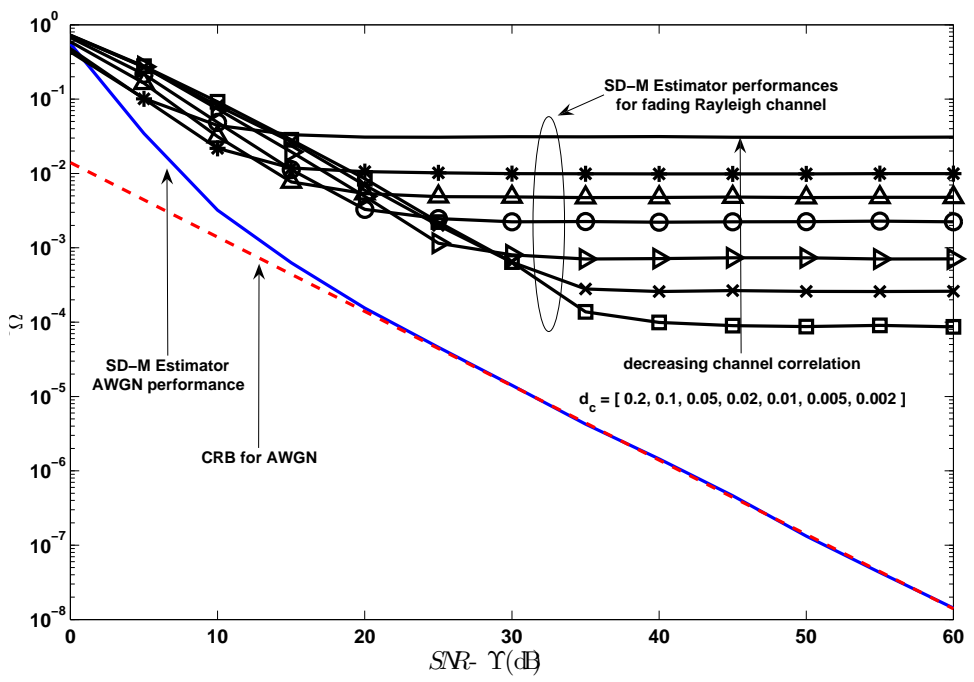

Fig. 5. Variance of the frequency estimates: $L=12, v=1.4, f_{s}=2 k H z$

varying channel conditions. Here, the Cramer-Rao lower bound [9] (for AWGN only channel) and the SD-M estimator performances for AWGN only channel are also shown together with the performances of the SD-M estimator for the flat fading noisy channel. Clearly, we see the differences in the performances when the channel undergoes fading, and especially when the channel becomes less time correlated (or fast flat fading). We also notice that the SD-M estimator variance reaches a threshold value when the $S N R$ is increased and remains unchanged when the $S N R$ is further increased, this is due to the same reason as discussed before where $S_{n}$ becomes more dominant than $\chi$. Threshold analysis for the estimator 
is important but is beyond the scope of this paper. The threshold effect becomes quite crucial especially when the channel is fast flat fading with more randomness in the channel, again in such scenarios increasing transmit signal power becomes useless. In such circumstances increasing $L$ is the way to improve jitter for a given channel.

\section{CONCLUSION}

The single-delay multiplication based frequency estimator was analysed for fast and slow flat fading channels. Analysis show that the estimator jitter performance depends on how well the channel is correlated in time. We present closed form solutions for the distribution of the frequency estimates for the flat fading channel and the variance of the frequency estimates. We also show how the estimator performance degrades when the fading becomes more random (less correlated). From the analysis we see that once the variance of the estimates reaches a threshold point increasing the transmit power does not improve the jitter performances any further.

\section{REFERENCES}

[1] Morelli, M.; Mengali, U.,"Carrier-frequency estimation for transmissions over selective channels" Communications, IEEE Transactions on Vol 48,Iss 9, Sept 2000 pp1580 - 1589

[2] W. Y. Kuo and M. P. Fitz, Frequency offset compensation of pilot symbol assisted modulation in frequency flat fading, IEEE Trans. Commun, vol. 45, pp.14121416, Nov. 1997.

[3] Chin Keong, Fung, P., "Using carrier frequency estimators developed for AWGN channel in multipath fading channel" ICASSP '03 IEEE Conference, Vol 6, 6-10 April 2003 pp:VI-565-8

[4] Lank G.W., Reed I.S., Pollon G.E., "A Semicoherent Detection and Doppler Estimation Statistic" Aerospace and Electronic Systems, IEEE Transactions on Vol AES-9,Iss 2, March 1973, pp:151-165

[5] Jackson. L, et al, "Frequency estimation by linear prediction”,IEEE Conference on ICASSP '78. Vol 3, Apr 1978 pp:352-356

[6] Kandeepan, S. and Reisenfeld, S. Analysis of a Discrete Complex Sinusoid Frequency Estimator Based on Single-Delay Multiplication Method, Int Symp on Information Theory, IEEE Conference on 'ISIT 05, 5-9 Sep, 2005, pp. 1451-1454.

[7] Handel P. and Volker B. , "Frequency estimation from proper sets of correlations"; Signal Processing, IEEE Transactions on, Vol 50,Iss 4,April 2002 pp:791 - 802

[8] S. Kay, "A Fast and Accurate Single Frequency Estimator," IEEE Transactions on Acoustics, Speech and Signal Proc, vol. 37 No.12, pp. 1987-1990, Dec 1989

[9] D. C. Rife and R. R. Boostyn, "Single-tone parameter estimation from discrete-time observations", IEEE Trans on Inform Theory, Vol IT,No 5,pp 591-598, Sep 1974.

[10] H. Meyr, M. Moeneclaey and S. A. Fechtel, Digital Communication receivers, Synchronisation, Channel Estimation and Signal Processing, John Wiley and Sons, 1998. 
[11] William H. Tranter et. al, Principles of Communication Systems Simulation with Wireless Applications, Prentice Hall, 2004.

[12] Anthanasios Papoulis, Probability, Random Variables, and Stochastic Processes, McGraw-Hill, 2nd Ed, New York 1984.

[13] S Kandeepan, Synchronisation techniques for digital modems, PhD thesis, University of Technology, Sydney, 2003

[14] S. Kandeepan, S. Reisenfeld, Performance Analysis of a Logarithmic Based Phase Detector for Tan-Locked Loops, IEEE conference on Personal Indoor Mobile and Radio Communications (PIMRC), Athens, 2007

[15] S. Kandeepan, Omar. H, Qian, A Complex Signal Based Digital Phase-Locked Loop with arctan Phase Detector Implemented on High Speed FPGA, IEEE conf on Info Comm \& Signal Processing 2007, Singapore

[16] S. Kandeepan, A Software Phase Locked Loop from Theory to Practice: TMS320C6000 DSP Based Implementation and Analysis, AusWireless conf 2006, Sydney March 13-16.

[17] S. Kandeepan, S. Reisenfeld. Frequency Error Correction for OFDM based Multicarrier Systems and Performance Analysis, IEEE Int Conf on Information, Communications and Signal Processing ICICS, 6-9 Dec, Bangkok, 2005.

[18] S. Kandeepan, S. Reisenfeld, Performance Analysis of a Digital Phase-Locked Loop with a Hyperbolic Nonlinearity, IEEE International Conference on Information, Communications and Signal Processing, 6-9 Dec, Bangkok, 2005.

[19] S. Kandeepan, and S. Reisenfeld, Acquisition Performance of a Digital Phase Locked Loop with a Four-Quadrant arcane based Phase Detector, IEEE International Symposium on Intelligent Signal Processing and Communications, Nov 2004.

[20] S. Kandeepan, and S. Reisenfeld, Fast Doppler Tracking DSP-Based Earth Station Modem for LEO Satellite Applications, IEEE Int Symp on Intelligent Signal Processing and Communications, Nov 2004

[21] S. Kandeepan, and S. Reisenfeld, Performance Analysis of DPLL with statistical filters for carrier synchronisation, IEEE International Conference on Signal Processing and Communications, Dec 2004.

[22] S. Kandeepan and S. Reisenfeld, Performance Analysis of a Correlator Based Maximum Likelihood Frequency Estimator, IEEE International Conference on Signal Processing and Communications, Dec 2004

[23] S. Kandeepan. and S. Reisenfeld, "DSP Based Frequency Estimation Techniques and Their Relative Performances", Symp on Communication Systems, Networks and Digital Signal Processing (CSNDSP2002), July 15-17, Staffordshire.

[24] S. Kandeepan and S. Reisenfeld, "Frequency Jitter of a Digital Phase-Locked Loop and comparison with a Modified CRB", IEEE International Conference on Communication Systems 2002, Nov 25-29. Singapore.

[25] S. Kandeepan and S. Reisenfeld, Phase detector models and their performances for IF/baseband frequency recovery for complex envelope based DSP implemented PLL, IEEE ICCS02, 25-28 Nov, 2002, Singapore

[26] S. Kandeepan and S. Reisenfeld, "A Complex Envelope Based 2nd Order Digital Phase Locked Loop and its Performance", IEEE International Symposium on Intelligent Signal Processing and Communication Systems 2001(ISPACS 2001) Proceedings of, Nov 20-23, Nashville

[27] S. Kandeepan, D. Jayalath, Narrow-Band Channel Simulator Based on Statistical Models, IEEE conference Communications and Signal Processing 2006, Singapore, Oct30-2Nov.

[28] Ramjee Prasad, OFDM for Wireless Communications Systems, Artech House, 2004 